\section{CMaP - A Collaborative Mapping Platform for Humanitarian Organizations}

Gl_Forum 2017, Issue 1

Page: 207 - 216

Short Paper

Corresponding Author: richard.schoerghofer@spatialservices.com

DOI: 10.1553/giscience2017_01_s207

\author{
Richard Schörghofer' ${ }^{1}$ Stefan Lang ${ }^{2}$, Lorenz Wendt² and Barbara Riedler ${ }^{2}$ \\ 'Spatial Services GmbH, Salzburg \\ 2Department of Geoinformatics - Z_GIS, University of Salzburg, Salzburg
}

\begin{abstract}
Within the EO4HumEn+ project (www.zgis.at/humanitarian-services), a collaborative web mapping platform was created, which not only collects the metadata of individual data sets created and enables searches on them, but also provides the functionalities to jointly create maps and other dynamic geospatial visualizations, and share them in collaborative tasks. The data in the web mapping platform can be accessed by read-only permission from all partners involved, and by full access permission for individuals who are entitled to either upload data or perform creative mapping - the so-called editors. A user manual guides editors through the three main steps of uploading, metadata creation and map production, by documenting the naming conventions and metadata requirements, as well as providing essential advice on data handling and mapping. The portal uses standardized formats, like metadata in ISO (International Organization for Standardization), Dublin or FGDC (Federal Geographic Data Committee), or external resources like Open Geospatial Consortium (OGC) Web Map Service (WMS), which offers standardized sharing capabilities via the services already mentioned. The Free and Open Source Software (FOSS) product GeoNode was chosen to implement an entry mask for the EO4HumEn+ data portal.
\end{abstract}

\title{
Keywords:
}

humanitarian mapping, EO products, metadata, standards

\section{Introduction}

The humanitarian community increasingly use Earth Observation (EO) data and its derived products and services to fill existing information gaps (Lang and Füreder, 2015). The project EO4HumEn+ (www.zgis.at/humanitarian-services) aims to extend the existing portfolio of established service tracks by developing and providing additional sub-services using EObased geospatial information for humanitarian action to international organizations and key players in the field. This endeavour strives to serve the diversifying needs of international humanitarian actors, including the International Committee of the Red Cross (ICRC), the Austrian Red Cross, SOS Children's Villages, Médecins Sans Frontières (MSF) and Groundwater Relief (GWR), for dedicated EO-derived information products. These 
products vary from population monitoring in camps and urban settings, to environmental resource monitoring including land use / land cover dynamics and (ground) water bodies, and assessment of societal vulnerability and hazard exposure. Information products will be provided using latest image analysis and geospatial data integration technologies as well as by adapting established workflows and tools.

To achieve these goals, one main strategic outcome of the project has been the development of a collaborative web mapping platform, called $\mathrm{CMaP}$. This platform not only collects the geospatial information products created along with their metadata, enabling searches on them, but also provides functionalities to jointly create maps and dynamic visualizations, and to share these in collaborative tasks.

$\mathrm{CMaP}$ uses standardized formats such as metadata that conform to ISO, Dublin or Federal Geographic Data Committee (FGDC); Open Geospatial Consortium (OGC) Web Map Service (WMS) is used for sharing data layers and including external sources. The Free and Open Source Software (FOSS) product GeoNode was chosen to implement the entry mask for the EO4HumEn+ mapping portal. To ensure the interoperability of the platform metadata portal with other catalogue services available on the web, the international ISO 19139 Metadata standard (ISO/TS, 2017) was chosen for the metadata to be created. CMaP is available at http:/ /eo4humen.sus4.eu/cmap.

Access to data uploaded to the platform can be granted to individual users, groups, or the general public. For searches on publicly-available data/metadata, no login is required. Search results are presented in a list. By default, a metadata record is presented in a 'simple view' which gives only abstract and contact information, but the section can be extended to show all entered attributes.

\section{Methods}

\section{Platform}

The platform is basically a combination of a metadata portal and a web mapping application. Metadata portals are commonly used for searching by standardized routines, applying an interchange format and semantics that allow these metadata to be queried also in other portals (Kuhn, 2005). Additionally, to provide desktop GIS-like functionalities the platform offers geodata visualization and simple spatial manipulation tools, such as editing, updating or deletion of point, line and polygon datasets. Other platforms offering these functionalities are GeoNode (2017) and Esri's ArcGIS Online ${ }^{\mathrm{TM}}$. GeoNode was chosen because it is opensource freeware and uses established metadata and geodata standards, which makes it interoperable with other platforms.

The technical implementation is in line with the standard web mapping three-tier architecture (Figure 1). It consists of a data / database tier, a (application) logical tier, and a presentation / user interface tier (Chen et al., 2003; Luqun et al., 2002). The data tier performs data storage, while the logical tier consists of a webserver and a GIS server. The presentation tier is used for visualization and includes an interface to other data tiers, which is adapted for specific purposes within the application context. 


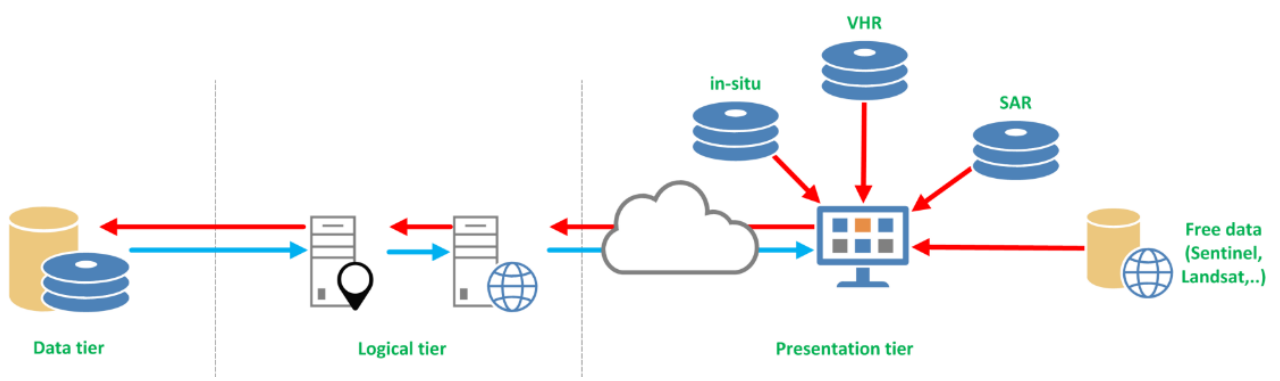

Figure 1: Three-tier web mapping architecture

For this project, a virtual server was used (acuGIS, 2017) configured with 150 GB of SSD storage, $10 \mathrm{~GB}$ of RAM, and 4 Core/3.4 GHz. The spatial data infrastructure (Nebert, 2004; Yue et al., 2015) for running the GeoNode operates on an Ubuntu Server 14.04.2 LTS (Ubuntu Server, 2017), where the installed software is a PostgreSQL DB 9.3 (PostgreSQL, 2017) with PostGIS 2.2 (PostGIS, 2017) for data storage; the webservers are an Apache Server (Apache HTTP Server, 2017), an Apache Tomcat ${ }^{\circledR}$ (Apache Tomcat, 2017), and a GeoServer 2.7.4 (special version for GeoNode) (GeoServer, 2017) as a GIS feature providing webserver and GeoNode 2.4.1. AcuGIS offers an all-in-one packaged GeoNode solution with all components preinstalled and is out-of-the-box runnable. With support included, this makes it cheaper than building your own setup of servers and GeoNode instances. A blank server with the same specs from Google or Amazon would have cost more than the whole package offered by acuGIS. ArcGIS Online (AGO) offers the same functionality as GeoNode, but it is not free, is not open source software (FOSS), and does not support the whole range of metadata and geodata standards to ensure interoperability with other platforms. 


\section{Layers / Documents / Maps}

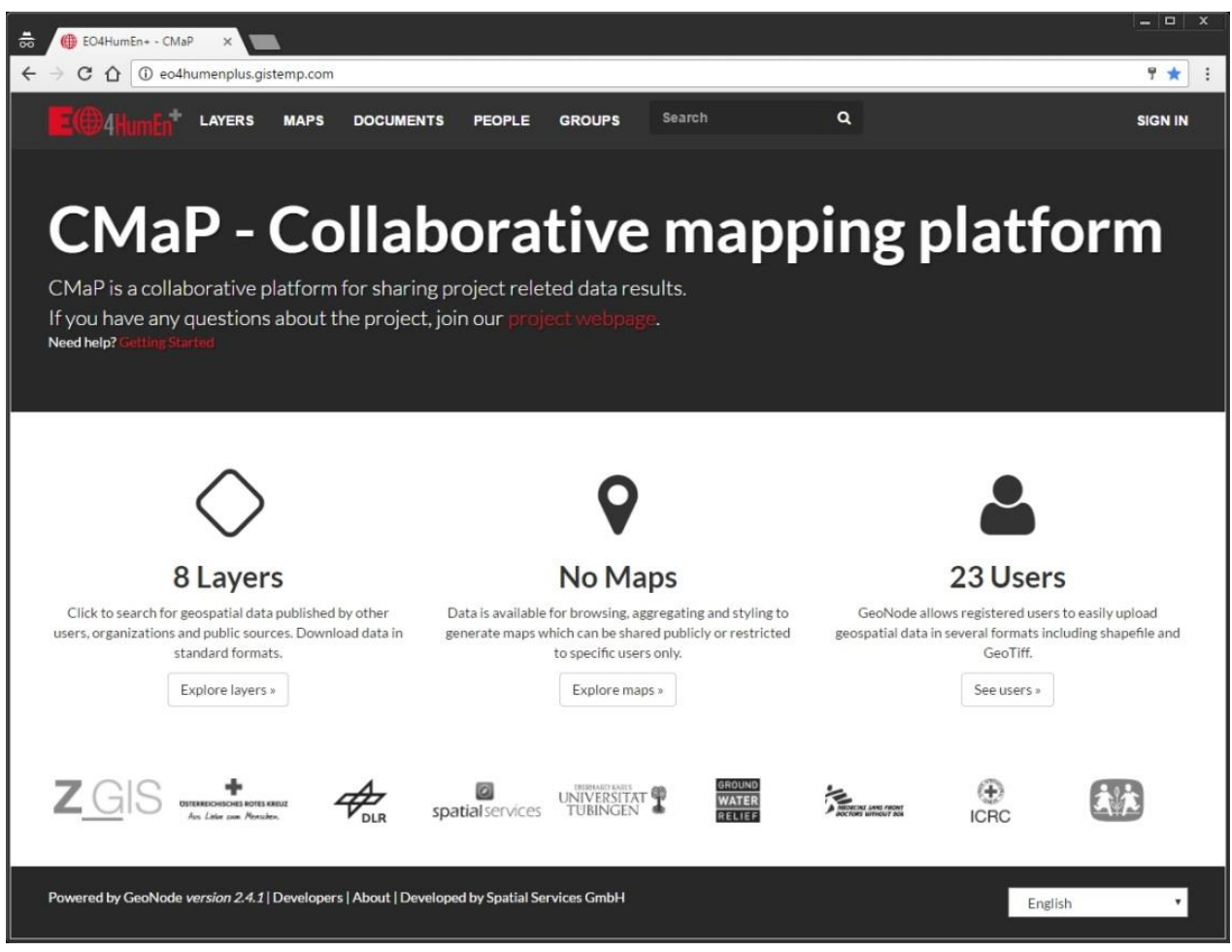

Figure 2: CMaP Entry mask

The generic GeoNode platform (Figure 2) has three main parts: (1) a section for spatial data named 'Layer', (2) a section for non-spatial data named 'Documents', and (3) a web mapping area called 'Maps'. The Layer section can handle vector and raster files. The file types are limited to shapefiles (.shp, .prj, .dbf and .shx) for vector files, and GeoTIFF for raster files. Simple editing is possible within the built-in GeoNode extension, GeoExplorer. Both file types can be individually styled (GeoServer SLD Cookbook, 2017) via the OGC SLD (Styled Layer Descriptor, 2017) styling.

The Document section has no limit to the types of file that can be uploaded, but it can be limited on demand. The documents can be linked to a layer file and receive the spatial extent from the layer file, allowing geospatial queries and searches for documents about specific localities.

External resources can also be embedded into the Layer or Document sections. Other WMS (Web Map Service, 2017), WFS (Web Feature Service, 2017) and WCS (Web Coverage Service, 2017), ArcGIS REST Services and Catalogue Services (Catalogue Service, 2017) can be embedded into the platform. Open Geoportal (OGP) and an adapted version of OGP (e.g. Harvard Geospatial Library) are offered as service-types as well. 
Maps are the third section of GeoNode. This is the web mapping platform to create individual maps using various layers from the Layer section. The created maps consist of a background layer and data layers (layer section). The background layer can be an external resource, or a predefined OpenLayers shortcut for background layers or Tile Map Service (TMS). The layers can be chosen from the layer section or from external resources such as WMS, WFS or other catalogue services. The styling of the layers can be individually chosen according to the purposes of the map, or use the default styling from the layer section.

\section{Metadata documentation}

Layers, documents and maps are saved along with metadata information. The mandatory metadata specification comprises title, abstract, category and keywords. Other information is inserted automatically from the login data, such as the timestamp from the server and spatial extension from the uploaded data itself. The automatic information can still be manipulated manually later, if required. The Documents use Dublin Core (DCMI, 2017); the Layer and Maps use ISO 19139 (ISO/TS, 2017) as standard.

In this project, the platform is used to store information products and data for the humanitarian community. The user must categorize the data and products as one of the four product families / categories (Table 1): Base data (DAT), for basic information such as administrative boundaries, satellite imagery etc.; Population (POP), for data and products relating to population numbers and densities; Environment (ENV), for data and products concerning environmental conditions, including land use/land cover, and groundwater; Resilience (RES), for data and products displaying the risk to and/or resilience against a given threat. Details of the categories are listed under 'Products' in Table 1.

Table 1: Product family types and product names

\begin{tabular}{|c|c|c|}
\hline Code & Product family & Products \\
\hline DAT & Baseline data & $\begin{array}{ll}\text { - } & \text { Digital elevation models } \\
\text { - } & \text { Synthetic Aperture Radar data } \\
\text { - } & \text { Optical satellite imagery } \\
\text { - } & \text { Reference data } \\
\text { - } & \text { Georeferenced thematic map } \\
\text { - } & \text { Situation map }\end{array}$ \\
\hline$\overline{\mathrm{POP}}$ & Population & $\begin{array}{ll}\text { - } & \text { Camp structure } \\
\text { - } & \text { Camp extent } \\
\text { - } & \text { Dwelling extraction } \\
\text { - } & \text { Dwelling density } \\
\text { - } & \text { Campelling change detection } \\
\text { - } & \text { Population estimation } \\
\text { - } & \text { Built-up structure } \\
\text { - } & \text { Built-up change detection } \\
\text { - } & \text { Graveyard detection } \\
\end{array}$ \\
\hline
\end{tabular}




\begin{tabular}{|l|l|ll|}
\hline & & $\bullet$ & Destroyed dwellings \\
\hline ENV & Environment & $\bullet$ & Hydrogeological reconnaissance map \\
& & - Riverbed analysis (jetting) \\
& & - Land use / land cover change \\
& & - Land use / land cover \\
& & - Habitat modelling \\
\hline RES & Resilience & $\bullet$ & Flood extent \\
& & - & Vulnerability \\
\hline
\end{tabular}

\section{User Management}

The user management of the web mapping platform discerns between two roles. While all partners involved in EO4HumEn+ have read access, entitled individuals have full access, allowing them to upload data and create maps. These users are called editors. A dedicated handbook guides editors through the three main topics (layers, maps and documents), explains the naming conventions and metadata requirements, and provides critical advice on data handling and mapping.

\section{Results}

The platform with all its components is already available (http://eo4humen.sus4.eu/cmap) and fully functioning (Figure 2). It requires little maintenance because of the well-developed open source software components on which GeoNode relies. The platform components are open-source, free of charge, and include all typical standards needed for interoperability with other metadata platforms; they also include web mapping standards from OGC. The mapping standards (like WMS) allow the building of self-designed stand-alone web mapping applications, similar to ArcGIS Online.

The results discussed here are for the example of Yumbe in Uganda, for which a groundwater overview map was created (Figure 3). In this case, the tool allows collaboration on the same map from various locations: a field team of geologists on site in Uganda, data analysts in England (GWR), and remote sensing experts in Salzburg (Z_GIS). In this way, a map with different layers, such as information regarding boreholes, a geological map, and a map of a planned refugee settlements, all from different sources, can be displayed and edited jointly. The tool allows all co-workers involved to access the same map, without each actor having to reproduce the map on his/her desktop GIS and share it later with the other actors. This simplifies the discussion of dispersed expert teams, helps avoid potential misunderstandings and production of errors, and allows for time-efficient working. The precise details of the development are based on actual user requirements as humanitarian actors are part of the project team, stimulating a rapid evaluation of newly implemented functions. Although designed as an online tool, maps can be printed if there is limited webaccess in the field. 


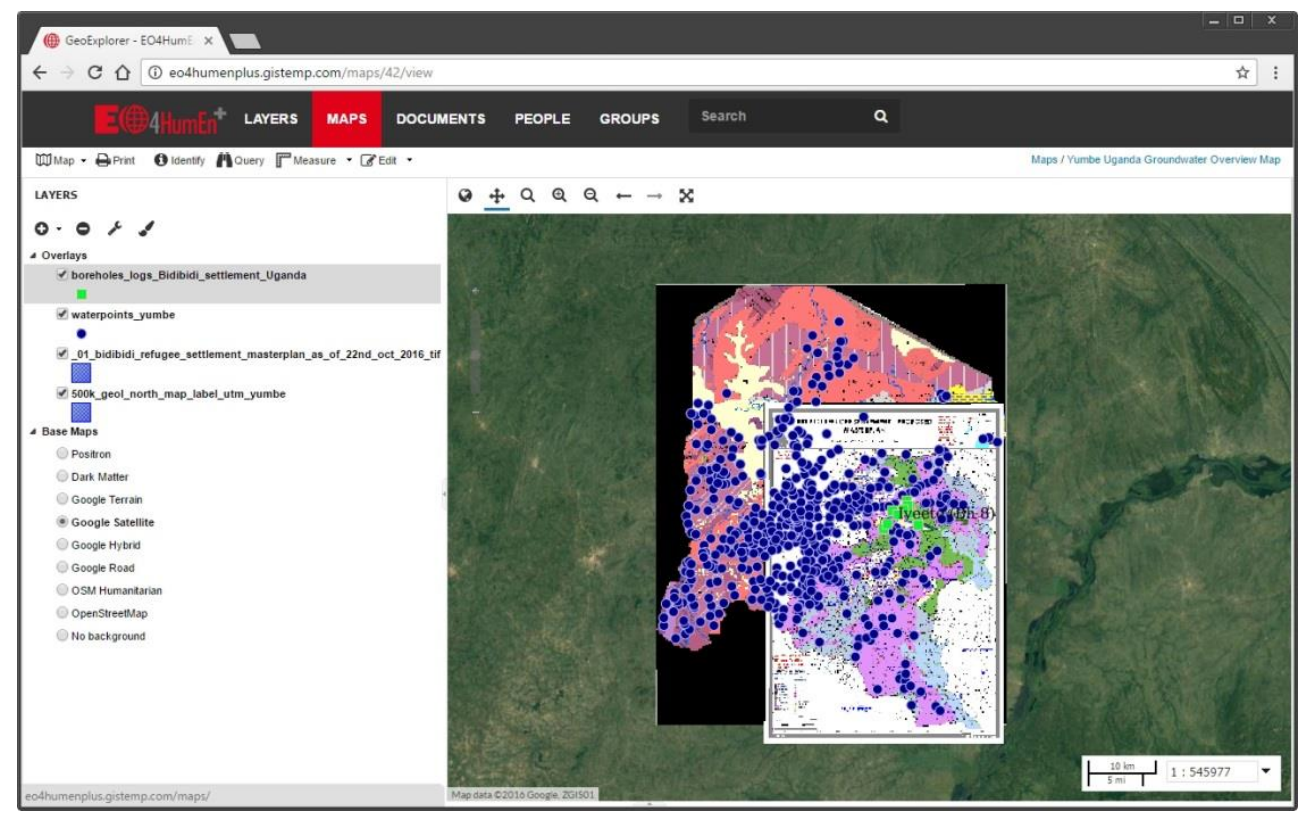

Figure 3: Groundwater Overview Map for Yumbe, Uganda

Another example of a map on this platform shows the refugee camp in Dagahaley, part of the Dadaab refugee camp complex in eastern Kenya, on the border with Somalia. In this case, the main purpose was not simply to demonstrate the collaborative aspect, but to test the capabilities for the production of informative and appealing web maps, accessible without GIS software or expertise. A very-high-resolution satellite image is streamed from another project-internal geospatial server such as WMS, and is tied into this map. The vector file presenting single dwellings extracted from the satellite image by an object-based image analysis is shown as an overlay in Figure 4, differentiating between different dwelling types according to a prior classification or categorization. In Figure 5, a dwelling density (raster) layer is shown on top of the VHR satellite image. 


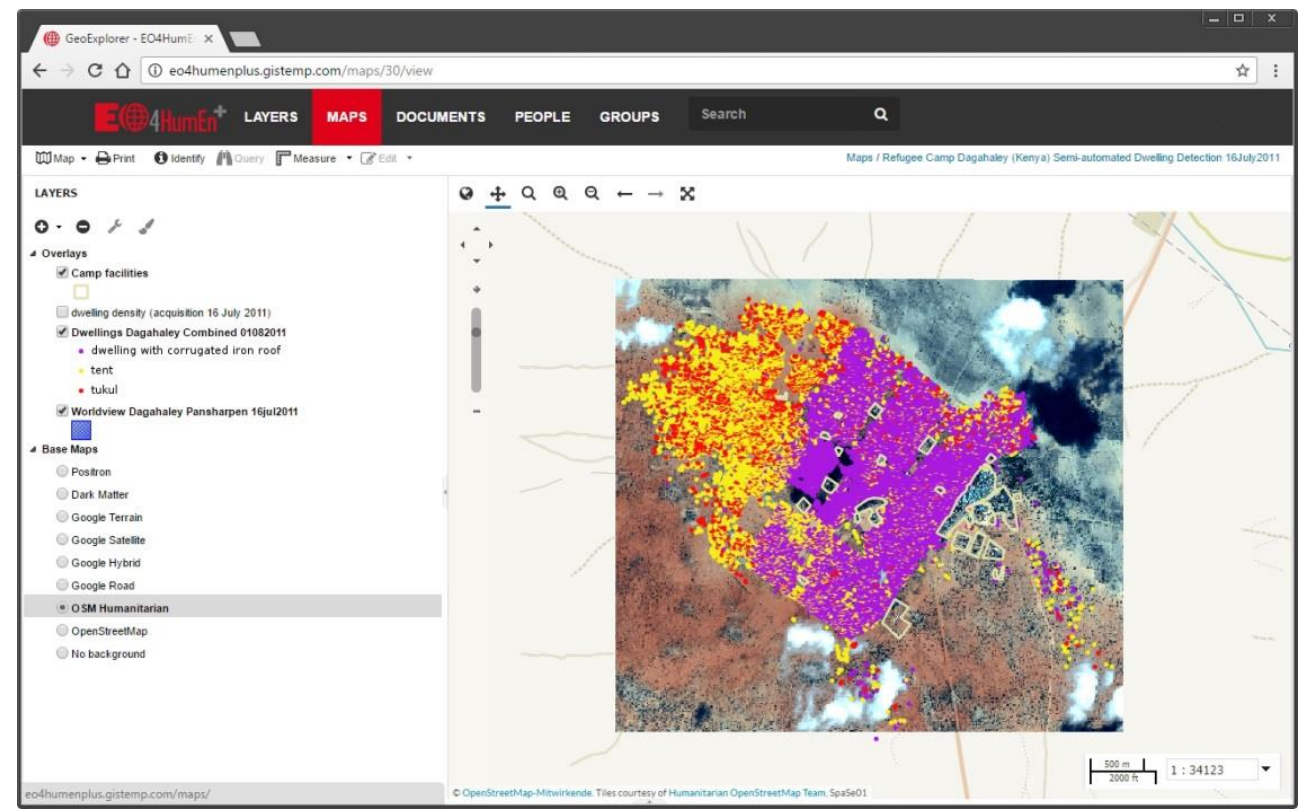

Figure 4: Dagahaley Refugee Camp (Kenya) - Semi-automated dwelling extraction, 16 July 2011

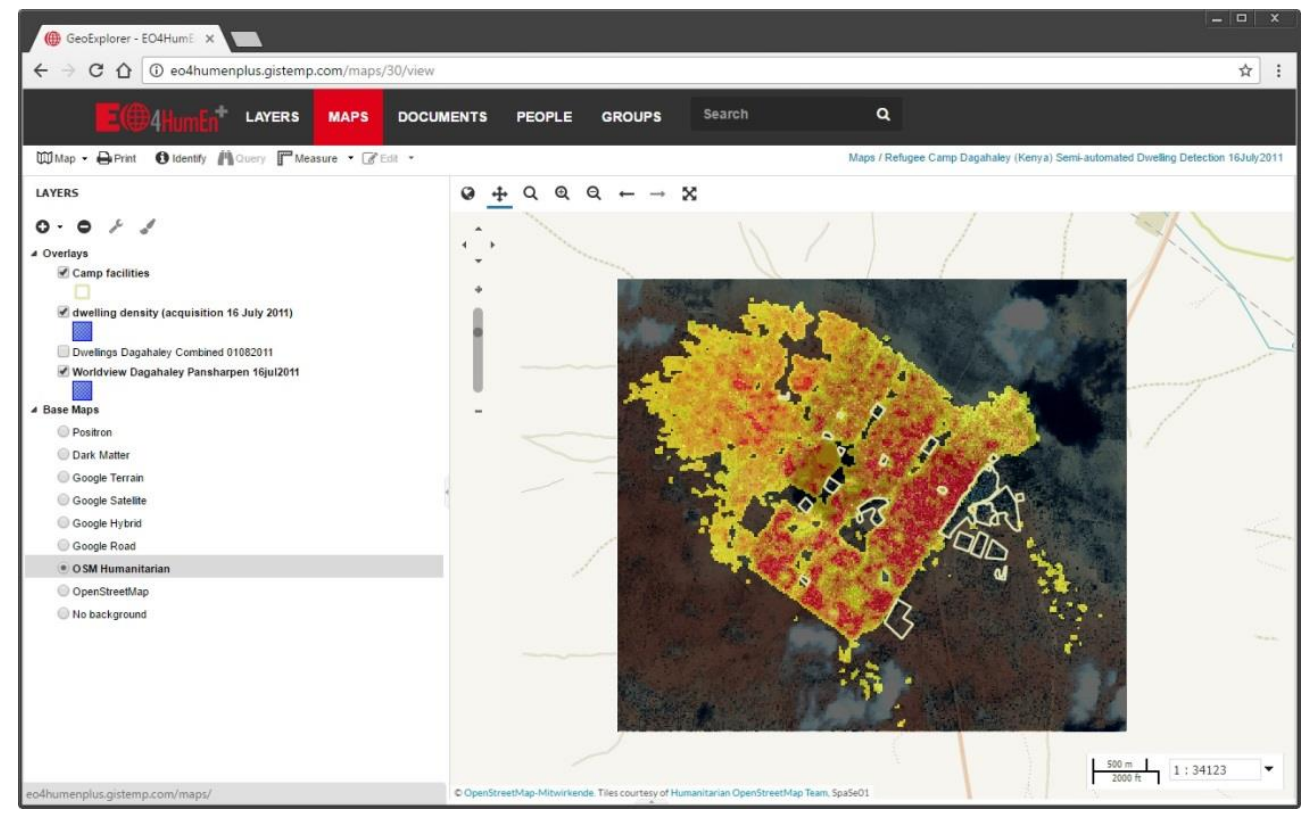

Figure 5: Dagahaley Refugee Camp (Kenya) - Population density based on semi-automated dwelling extraction, 16 July 2011 


\section{Discussion and Outlook}

While saving data in a spatial data infrastructure (SDI) (Nebert, 2004; Yue et al., 2015) solution like GeoNode is a well-established routine, the combination of a metadata portal with web mapping techniques is a new way for different partners to collaborate in sharing data and products on a centralized platform (Granell et al., 2014).

The platform needs further development by the open-source community because it is not currently possible to use vector formats other than shapefiles such as KML and JSON, or raster formats other than TIFF. The platform deals with these restrictions by generating warning messages. The uploading process is sensitive and requires well formatted datasets; and, depending on the file size, it can take a while for the data to be uploaded and become available.

The procedure for uploading documents is, however, straightforward, and the uploading chain is simple. During a test phase where experts from the EO4HumEn+ project tested the platform, no errors occurred when data were submitted to the platform.

The map area tab in GeoNode is self-explanatory. Maps are produced as a combination of various layers. Unlike AGO, which not only decouples the map from the portal but also offers templates for individual designs, the platform does not offer the possibility of using maps in predefined stand-alone web mapping applications without the platform.

$\mathrm{CMaP}$ is an exploratory playground within the project EO4HumEn+ to be developed further into an interactive platform, which can be used by the humanitarian organizations involved. Users can share information directly in the platform, use the datasets, edit input layers and jointly produce and design custom-made information products. It will be tested throughout the project's lifetime for its potential for effective collaborative mapping in realworld scenarios.

\section{Acknowledgements}

This work has been co-funded by the Austrian Research Promotion Agency (FFG) under the Austrian Space Application Programme (ASAP) within the project EO4HumEn+ (contract no: 854041).

We would like to thank Médecins Sans Frontières (MSF), especially Edith Rogenhofer from MSF Austria, and the GSI unit in Geneva for sharing data. 


\section{References}

acuGIS GeoNode Cloud Hosting. (2017, January 26). Retrieved from

https://www.acugis.com/geonode-hosting.html

Apache HTTP Server. (2017, January 26). Retrieved from https://httpd.apache.org/

Apache Tomcat ${ }^{\circledR}$. (2017, January 26). Retrieved from http://tomcat.apache.org/

Catalogue Service. (2017, January 26). Retrieved from http://www.opengeospatial.org/standards/cat

Chen, S., Gulati, S., Hamid, S., Huang, X., Luo, L., Morisseau-Leroy, N., Powell, M.D., Zhan, C., \& Zhang, C. (2003). A Three-tier System Architecture Design and Development for Hurricane Occurrence Simulation.

DCMI. (2017, January 26). Retrieved from http://dublincore.org/

GeoNode - Open Source Geospatial Content Management System. (2017, January 26). Retrieved from http://geonode.org/

GeoServer is an open source server for sharing geospatial data. (2017, January 26). Retrieved from http://geoserver.org/

GeoServer SLD Cookbook. (2017, March 17). Retrieved from http://docs.geoserver.org/stable/en/user/styling/sld/cookbook/

Granell, C., Fernández, Ó., Díaz, L. (2014). Geospatial information infrastructures to address spatial needs in health: Collaboration, challenges and opportunities, Future Generation Computer Systems, 31, 213-222, ISSN 0167-739X, http://dx.doi.org/10.1016/j.future.2013.04.002.

ISO/TS 19139:2007. (2017, January 26). Retrieved from http://www.iso.org/iso/catalogue_detail.htm?csnumber=32557

Kuhn, W. (2005). Geospatial Semantics: Why, of What, and How? Journal on Data Semantics III, LNCS 3534, $1-24$.

Lang, S., \& Füreder, P. (2015). Earth Observation for Humanitarian Operations. 2015. In: Car, A., Jekel, T., Strobl, J., Griesebner, G., (Eds.) GI_Forum_2015 - Geospatial Minds for Society, 2015, 384-390.

Luqun, L., Jian, L., \& Yu, T. (2002). The Study on Web Gis Architecture Based on Jnlp.

Nebert, D. (Ed.) (2004), The SDI Cookbook. Global Spatial Data Infrastructure Association.

Retrieved from http://gsdiassociation.org/index.php/publications/sdi-cookbooks.html.

PostGIS. (2017, January 26). Retrieved from http://www.postgis.net/

PostgreSQL. (2017, January 26). Retrieved from https://www.postgresql.org/

Styled Layer Descriptor. (2017, January 26). Retrieved from http://www.opengeospatial.org/standards/sld

Ubuntu Server. (2017, January 26). Retrieved from https://www.ubuntu.com/server

Web Coverage Service. (2017, January 26). Retrieved from http://www.opengeospatial.org/standards/wcs

Web Feature Service. (2017, January 26). Retrieved from http://www.opengeospatial.org/standards/wfs

Web Map Service. (2017, January 26). Retrieved from http://www.opengeospatial.org/standards/wms

Yue, P., Baumann, P., Bugbee, K., \& Jiang, L. (2015). Towards intelligent GIServices. Earth Sci. Inform., 8 (3), 463-481. 